\title{
Laser Sounder for Active Remote Sensing Measurements of $\mathrm{CO}_{2}$ Concentrations.
}

\author{
Graham R. Allan ${ }^{1}$, Haris Riris ${ }^{2}$, James B. Abshire ${ }^{2}$, Xiaoli Sun ${ }^{2}$, Emily Wilson ${ }^{3}$, John F Burris ${ }^{2}$ \& Michael A. Krainak ${ }^{3}$ \\ (1) - Sigma Space Inc., NASA GSFC, Mail Code 694, Greenbelt MD 20771USA \\ (2) -NASA Goddard Space Flight Center, Mail Code 694, Greenbelt MD 20771 USA \\ (3) -NASA Goddard Space Flight Center, Laser and Electro-Optics Branch, Code 554, Greenbelt MD 20771 USA \\ graham.allan@gsfc.nasa.gov
}

Abstract-We report on progress of our $\mathrm{CO}_{2}$ laser sounder laboratory breadboard system the goal of which is to measure the integrated column abundance of $\mathrm{CO}_{2}$ to better than $1 \mathrm{ppm}$ from low Earth orbit globally, measuring at all latitudes and seasons through day and night. The challenge for an orbiting $\mathrm{CO}_{2}$ instrument is to achieve high precision not high sensitivity. We have made simple yet significant improvements to our active, optical-sensing laser-sounder instrument and real-time data processing that now enables absolute absorption measurements to better than $\pm 0.05 \%$ for over 10 hours before re-calibration (equivalent to a $1 \mathrm{ppm}$ precision from orbit). Data from an eight day, $0.8 \mathrm{Km}$ open path comparison test with a LICOR shows excellent agreement. ${ }^{12}$

\section{TABLE OF CONTENTS}

1. INTRODUCTION ....................................................... 1

2. INSTRUMENT DESCRIPTION .................................. 2

3. MEASUREMENTS.................................................. 4

4. SUMMARY …........................................................... 7

REFERENCES …............................................................ 7

BIOGRAPHY................................................................ 7

\section{INTRODUCTION}

Accurate global measurements of $\mathrm{CO}_{2}$ mixing ratios is of great scientific and popular interest and yet there is substantial uncertainty in the current atmospheric $\mathrm{CO}_{2}$ budget. The techniques for measuring $\mathrm{CO}_{2}$ are spatially localized and quite antiquated. Currently, the most comprehensive set of atmospheric $\mathrm{CO}_{2}$ data is from the National Oceanic and Atmospheric Administration (NOAA) Climate Monitoring and Diagnostics Laboratory (CMDL) cooperative air-sampling network, consisting of more than 40 sites where flasks of air are collected approximately weekly and analyzed. CMDL reports that the average annual increase in $\mathrm{CO}_{2}$ mixing ratio, since 1980 , has been on the order of $1 \mathrm{ppm} / \mathrm{year}$ and the average $\mathrm{CO}_{2}$ mixing ratio currently stands at approximately 382 $\mathrm{ppm}$. The continued addition of anthropogenic $\mathrm{CO}_{2}$ to the naturally occurring $\mathrm{CO}_{2}$ background strongly suggests that present levels of $\mathrm{CO}_{2}$ should be much higher and that an unknown sink is responsible for the present levels. Thus, any instrument attempting to measure $\mathrm{CO}_{2}$ mixing ratios

\footnotetext{
${ }^{1}$ U.S. Government work not protected by U.S. copyright
}

${ }^{2}$ IEEEAC paper\#1257, Version 4, Updated 2007:10:25 from space and identify the missing sink must be able to measure the absolute mixing ratio with a long-term precision of $1 \mathrm{ppm}$ and be able to measure globally to all latitudes, in all seasons measuring over both day and night. The current state of the art for passive space-based remote sensing of atmospheric $\mathrm{CO}_{2}$ is the Orbiting Carbon Observatory (OCO), scheduled for launch in 2008. OCO is a NASA mission for measuring total column $\mathrm{CO}_{2}$ and $\mathrm{O}_{2}$ by detecting absorption in reflected sunlight [1]. Although

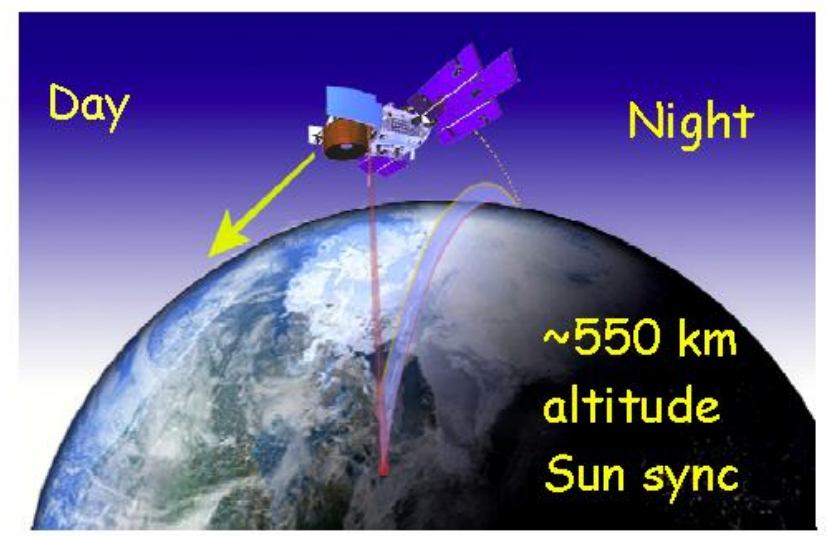

Figure 1. A near-polar orbiting active laser sounder instrument can "unambiguously" globally map the $\mathrm{CO}_{2}$ mixing ratios at high and low latitudes, in all seasons and in day or night conditions.

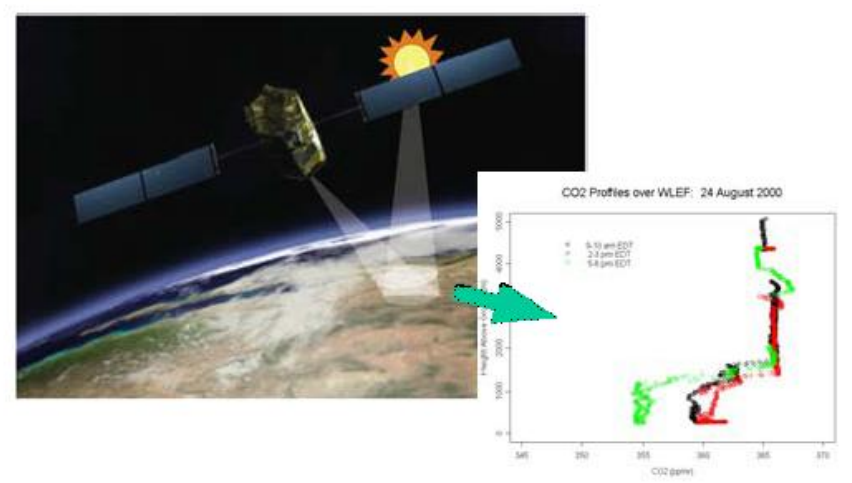

Figure 2. The Orbiting Carb on Observatory has unavoidable physical limitations imposed by the passive measurement approach that include: daytime/high sun only sampling, interference by cloud and aerosol scattering, non-uniform $\mathrm{CO}_{2}$ profile and limited signal variability in the $\mathrm{CO} 2$ column

OCO will yield important new information about the distribution of atmospheric $\mathrm{CO}_{2}$, there are unavoidable 
physical limitations imposed by the passive measurement approach that include: daytime/high sun only sampling, interference by cloud and aerosol scattering, and limited signal variability in the $\mathrm{CO}_{2}$ column. See figures $1 \& 2$. There is a critical need for an active laser remote sensing instrument that can measure with $1 \mathrm{ppm}$ accuracy from space which will be able to answer the question: where is all the $\mathrm{CO}_{2}$ going? In this paper we report on progress and results from our breadboard, active, optical-sensing system in measuring open-path, integrated-column abundance of $\mathrm{CO}_{2}$ in real time. We have made simple yet significant improvements to our bread-board laser-sounder instrument and real-time data processing that now enables absolute absorption measurements to better than $\pm 0.05 \%$. The transmitter is a tunable, narrow-linewidth $(\mathrm{MHz})$ diodelaser seeded $0.5 \mathrm{~W}$ Erbium Doped Fiber Amplifier in the Master Oscillator Power Amplifier configuration, operating in the $1572 \mathrm{~nm} \mathrm{CO} 2$ absorption band in pulsed or chopped cw-mode. The receiver consists of a modified 8" SchmidtCassegrain telescope fiber coupled (multi-mode) to either a InGaAs pin diode or photomultiplier tube. The system is light weight, efficient and easily scalable to higher optical powers. The frequency scanning system measures the line shape, removes background structure and instrument effects and determines the integrated column abundance of $\mathrm{CO}_{2}$.

All optical systems that measure absolute absorption are subject to systematic errors that arise from interference effects, dispersion, frequency dependent gain and reflection losses, all of which introduce structure, easily mistaken for the desired signal. These frequency dependent noise sources are time varying and difficult to eliminate. Our system, like most, is designed to minimize these effects, however any error in determining the background greatly affects the absorption accuracy. We have modified both our hardware and software that in real-time tracts the noise and background structure and minimizes these effect on the data. The addition of a wedged beam splitter and integrating sphere to the transmitter power monitor minimizes the introduction of any interference effects and enables reliable ratio-ing of the signal and monitor and better determination of the background structure. We improve on the standard on-line off-line determination of absorption since we directly measure the absorption line shape. In this paper we present data on stability and open path measurements of $\mathrm{CO}_{2}$. The zeropath measurements show an accuracy of better than $\pm 0.05 \%$ over 10 hours without re-calibration. Data comparisons with a spot measurements instrument (LICOR) shows correlation with the diurnal cycle and rush-hour variations in $\mathrm{CO}_{2}$. We will show data from $0.4 \mathrm{~km}$ one way open path to a cooperative target using an InGaAs pin diode and from uncooperative target using photon-counting PMT. In addition we will present data from our upcoming Fall-2007 field test comparison with calibrated instruments on the NOAABAO tower in Erie, Colorado.

\section{INSTRUMENT DESCRIPTION}

The laser sounding technique measures the total column average absorption by $\mathrm{CO}_{2}$ from space. A co-located measurements of the $\mathrm{O} 2$ column absorption will allow us to account for variations in the $\mathrm{CO}_{2}$ absorption path caused by topography, surface pressure changes, and variable humidity (as well as having major independent benefit to weather analysis and forecasting). Our method can measure at all latitudes at any sun angle, e.g., dawn and dusk, and is more robust against bias caused by diurnal variations in surface $\mathrm{CO}_{2}$ mixing ratios.

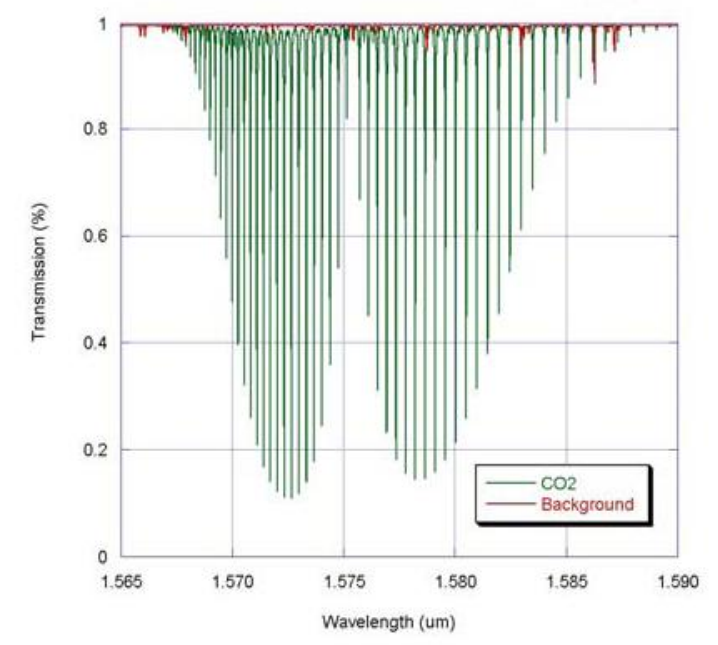

Figure 3. Near IR $\mathrm{CO}_{2}$ and background spectra from space.

We have selected the spectral region near $1.57 \mu \mathrm{m}$ based on several criteria. The spectral band consists of discrete lines that are free from interference from water vapor and other trace atmospheric gases. The optical depths are strong enough to provide high sensitivity to changes in the $\mathrm{CO} 2$ column. In addition, this wavelength falls within the telecommunications "L-Band" and it is also a wavelength region where the defense and security industry is also interested for various applications. Our design is synergistic with the technology advancements in this area and we can leverage the substantial commercial and government research and development effort focused on improving components in this wavelength region. High power erbium doped fiber amplifiers (EDFA), used in long haul telecommunication networks, are rugged, fairly compact, and extremely reliable. They have achieved powers of several hundreds of Watts. Their spectroscopic properties (narrow linewidth and high spectral purity) make them suitable for this application. They can also be space qualified as more defense and security applications are implemented. In addition, single-frequency, narrow linewidth, distributed feedback (DFB) semiconductor lasers are available for the $1.57 \mu \mathrm{m}$ transmitter. These low power lasers will seed the EDFAs to generate the high powers needed for operation from orbit. Receiver components 
(detectors) are also commercially available at this wavelength.

Although the $\mathrm{CO}_{2}$ absorption band near $1570 \mathrm{~nm}$ is relatively free from interferences by other species (Figure 3 ) it is crucial that the most suitable line is selected for the sounder approach. The spectroscopic conditions that must be met (low temperature sensitivity, negligible interferences from other species, etc.) are analyzed in detail by Mao et.al. [2].

Traditional DIAL uses only two lines (on and off) to measure the absorption. Our technique will use six or more lasers to sample the $\mathrm{CO}_{2}$ absorption line. By judiciously choosing the wavelength of each laser line we can weigh the measurements to the lowest part of the troposphere where most of the $\mathrm{CO}_{2}$ signal variability occurs.

The wavelength of each laser must be precisely controlled to a high degree of accuracy and must be actively locked to the wavelength of interest. The most stringent requirement applies to the lasers locked to the side of the absorption where the slope is the highest and the least stringent to the peak and wings of the absorption where the slope is the lowest. For a pressure broadened absorption, the locking requirement is $2 \mathrm{MHz}$ for the lasers locked on the side of the absorption and roughly $30 \mathrm{MHz}$ for the peak. For the initial breadboard work we use a frequency tunable diode laser that substitutes for multiple lasers.

Precise, absolute spectroscopic measurements of trace gases present enormous challenges to any instrument (ground or space-borne). Typically, a laser absorption spectrometer based on a direct absorption technique is capable of measuring absorptions in the $10^{-2}$ to $10^{-3}$ range with a $1 \mathrm{sec}$ averaging time. High frequency modulation techniques such as frequency and wavelength modulation have increased the sensitivity limit to $10^{-7}$ to $10^{-8}$ (the quantum or shot noise limit) using phase sensitive detection and various modulation schemes. Phase sensitive detection techniques achieve their high sensitivity by detecting in a region of the frequency spectrum where there is little $1 / \mathrm{f}$ noise (most lasers and detectors exhibit $1 /$ f or flicker noise). However, the challenge for a $\mathrm{CO}_{2}$ instrument is to achieve high precision (or stability) not high sensitivity. The absorption from space is roughly $90 \%$ so there is no need for high sensitivity. The challenge of obtaining 1-ppm long-term precision (or stability) is very different from obtaining high sensitivity. The metrics used for long-term precision (stability) and sensitivity, are also different. High signal to noise (SNR), traditionally used to denote the sensitivity of a spectrometer in a $1-\mathrm{Hz}$ bandwidth, is not a good metric in describing the long-term precision. Achieving a high SNR with a $1 \mathrm{sec}$ averaging time does not imply a long-term precision capability for arbitrarily long times.

In fact it has been shown that longer averaging times can actually degrade the SNR. Increasing the averaging time will reduce random noise, but non-random "noise" sources, typically described as "drifts" in the system, will actually degrade the ability of the system to discern small changes in absorption.

These issues were recognized and addressed in frequency modulation spectrometers (see for example Werle et.al. [3]), but they are applicable to any laser spectrometer, independent of detection method. In order to address them in a more quantitative fashion and determine the maximum averaging time for a spectrometer Werle et.al. used the Allan variance as a metric of the system's stability as opposed to the SNR.

There are many sources of noise and "drifts" in a laser spectrometer. Examples of random noise include thermal (or Johnson) noise, shot (quantum) noise, and detector noise. Examples of "drifts" or non-random noise include opto-mechanical drifts due to temperature changes, etalon fringes, laser wavelength drift etc. All of these noise sources and drifts contribute to the total error budget of the instrument. One of the most difficult error sources to address is etalon fringes. Etalon fringes are interference patterns generated by multiple reflections in the optical path and small transmission variations in optical elements, which result in a background structure that is time dependent and changes as a function of temperature, pressure, vibration, or mechanical stress. More often than not this time-varying background has a similar signature as the absorption feature of interest making it exceedingly difficult to suppress. This background structure is present in all absorption spectrometers independent of detection method (direct detection, phase sensitive detection, DIAL, etc.) or type of detector used and cannot be eliminated by simple filtering. If etalons were stationary in time they could be subtracted and would simply represent an offset in the total absorption measurement. However, since they vary on time scales comparable to the signal of interest (i.e. $\mathrm{CO} 2$ absorption) they will be interpreted as signal as their amplitude and/or phase changes.

The challenge of suppressing etalon fringes was recognized decades ago and many hardware and software methods have been successful in reducing, but not totally eliminating, their impact [4-7]. Careful optical design is of paramount importance. Anti-reflection coatings, the use of reflective as opposed to transmissive optics, angled or wedged optics can keep etalon fringes to a minimum. Frequent calibrations for in-situ measurements in cells are also used to minimize the effect of drifts. Unfortunately this is not practical in a space mission.

Most hardware techniques try to "wash out" the fringes (i.e. reduce their coherence) by vibrating an optical element (a mirror or Brewster plate). This can be effective if there is only one dominant etalon fringe whose structure does not change significantly over time. This approach would not be practical in a space instrument since we cannot fully characterize or have prior knowledge of the instrument 
drifts in space. In addition, it would add cost and complexity to the instrument.

Most software methods use digital signal processing to filter out the fringes. Digital filters $([8,9])$ can be effective in reducing the effect of fringes however they do have their limitations. The unwanted background structure usually includes several fringes with different dependence on temperature, pressure, and other factors. The fringe free spectral range (or period) may be large making it difficult to determine its frequency and phase and devise an effective filter. Finally some fringes will have the same or comparable free spectral range as the width of the absorption making them virtually impossible to filter without filtering out the absorption signal. Despite these limitations, signal processing because of its flexibility, low cost, and zero mass remains the preferred solution for a space mission.

We have used a combination of hardware and software techniques to reduce the effect of fringes and other drifts in our $\mathrm{CO}_{2}$ sensor. The present breadboard configuration consists of a distributed-feedback diode laser operating at $1572.33 \mathrm{~nm}$, which acts as a seed for a polarization maintaining $500 \mathrm{~mW}$ EDFA. A single laser was used in the long-term precision (stability) experiments. We have successfully used a two-laser approach and have been successful in locking the laser wavelength within $12 \mathrm{MHz}$ using a commercial wavemeter. However, in order to demonstrate the required stability a single laser was used. A small percentage of the seed laser is directed into a fiber cell containing $\mathrm{CO}_{2}$ for wavelength calibration purposes, figure 4. The laser wavelength is scanned over the absorption using a small ramp from a waveform generator. The amplified seed from the EDFA is collimated in free space and about $10 \%$ is directed into an integrating-sphere power-monitor assembly to normalize output power fluctuations of the transmitter and any common mode noise and fringes. The rest of the beam is directed outside our laboratory onto a hard or soft target (trees) depending on the type of detector we are using (a photodiode or a PMT can be used with the system). The return is collected by an all-reflective 8" diameter Schmidt-Cassegrain telescope and coupled into a large core high NA fiber. The fiber is then connected to either a photomultiplier tube or a photodiode depending on the experiment. The path length to the hard target is approximately $400 \mathrm{~m}$. A mechanical chopper modulates the beam to establish a zero-baseline offset and subtract any variations in the baseline due to the solar background or other offsets.

A computer digitizes the signals, divides the signal by the reference and applies a real-time signal-processing algorithm (similar to a matched filter) to estimate the size of absorption. The transmitter (and telescope) can be turned 180 degrees and the beam directed through an absorption cell and onto a diffuse target. The receiver telescope then collects the light scattered by the diffuse target.

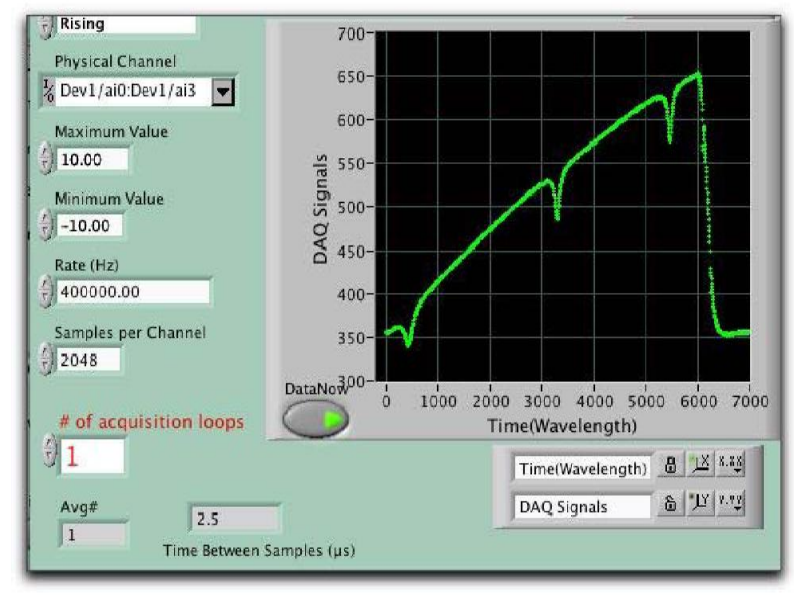

Figure 4. Plot of laser power as a function of drive current recorded after an absorption cell. As the laser is current-tuned the output power increases and sweeps through three absorptions. The center absorption is the $1572.33 \mathrm{~nm}$ CO2 line.

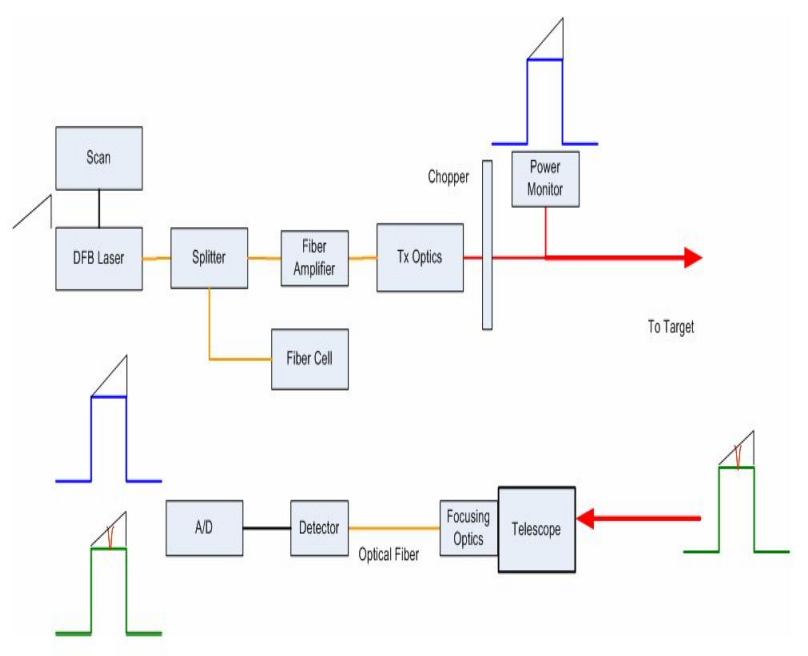

Figure 5. Breadboard functional block diagram showing the transmitter (upper) and receiver (lower).

\section{Measurements}

\section{Zero-path measurement stability measurements.}

To evaluate the long-term precision of our transmitter we measure the absorption through our "zero-path" setup. This consists of an absorption cell containing a known concentration of $\mathrm{CO}_{2}$, several folding optics and a scattering surface. The co-aligned transmitter/receiver scope is rotated from the open path test range to point at the absorption cell. 


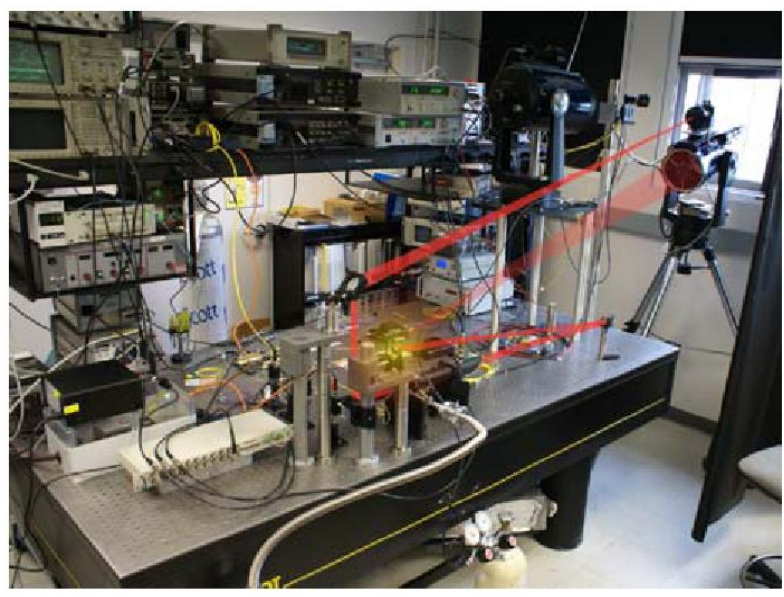

Figure 6. Photograph of the zero-path setup. The coaligned transmitter/receiver scope is rotated from the open path test range to point at the absorption cell. The

transmitted light is directed to a scattering screen viewed by the receiver scope without need to refocus or adjust boresight.

The unabsorbed light is directed to a scattering screen which is viewed by the receiver scope without need of refocusing or re-alignment. The cell is setup so that during our open path measurements the transmitter/receiver can be turned around and checked against our "zero-path" reference. To distinguish the in-cell measurements from the open-path range we refer to the in-cell measurements as "zero-path", the actual optical path through the cell is $0.33 \mathrm{~m}$. The cell is filled with approximately 10 mbar of pure $(99.99 \%) \mathrm{CO}_{2}$ and the peak absorption measured as a function of time. Figure 7 is a time series of data over approximately 60 hours. Over this period the cell pressure slowly increases due to a slow-leak to air. The line is thus buffered by increasing air pressure. The natural abundance of $\mathrm{CO}_{2}$ in atmospheric air entering the cell is too low to affect the measurement or in the minimal open-air optical path in the "zero-path" setup. The decrease in absorption with time is due to pressure broadening and is compensated by an increasing linewidth (not shown). The solid red line is a the linear fit through the data. The long-term precision, as determined by the min-max (not the standard deviation or the Allan variance) of the entire time series data is an absorption of $0.1 \%$, although there are periods where the system is much more stable. Figure 8 plots the difference between the measured peak absorption and the linear fit shown in figure 7 . For over 12 hours the measured reading is within $\pm 0.05 \%$ of the peak absorption as determined from the linear fit.

Depending on the selection of the $\mathrm{CO}_{2}$ line we can expect a $50-90 \%$ absorption assuming a mixing ratio of $370-380$ ppm. Using the $50 \%$ absorption from space our long term precision translates to $0.13 \%$. In order to meet the $1-\mathrm{ppm}$ requirement a $1 / 370$ or $0.27 \%$ precision would be needed. One cannot expect any system to remain stable indefinitely, especially without any cross checks or calibration. This system exceeds the necessary long-term precision required for a space based instrument.

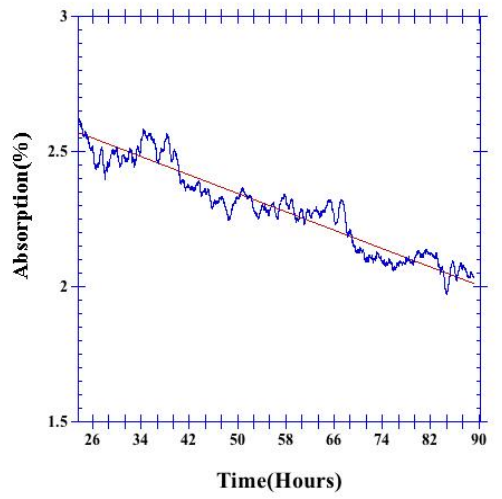

Figure 7. Measured absorption in the "Zero-path" setup as a function of time. The slow decrease in absorption is due to pressure-broadening. The cell initially contained $\sim \mathbf{1 0}$ mbar of pure $\mathrm{CO} 2$. Atmospheric air, slow leak, increased the pressure, broadening the line and decreasing the peak absorption.

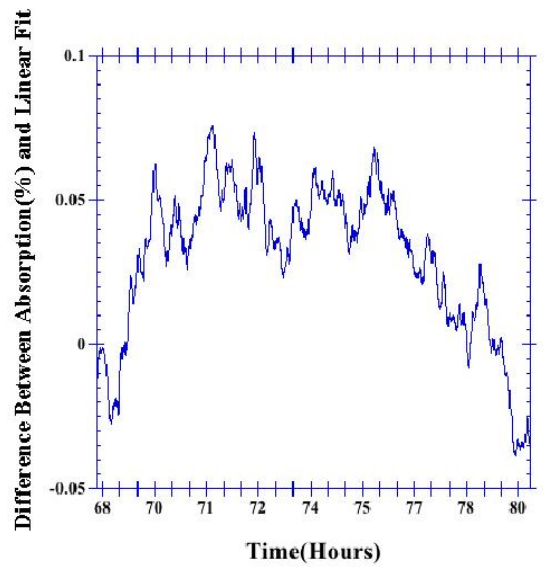

Figure 8. Difference between measured reading and fit over a 12 hour period shown in figure 7. Data shows a region where we meet the $\pm 0.05 \%$ spec for $\sim 12$ hours.

From space we have an opportunity to use measurements of $\mathrm{CO} 2$ over the Antarctic continent as a cross check. The $\mathrm{CO} 2$ over Antarctica is essentially constant through out the year. Assuming an orbital period of approximately 90 minutes the system will have several passes to perform a necessary calibration cross check. Our breadboard sensor meets the long-term precision for well over 90 minutes.

A $\mathrm{CO} 2$ sensor not only needs long-term precision but it needs to measure the absolute $\mathrm{CO}_{2}$ mixing ratio. We compared our breadboard system with a $\mathrm{CO}_{2}$ measurement standard (LICOR CO2 / 20 gas analyzer).

The LICOR gas analyzer draws air from the top of the building above where our breadboard system is located, so it is a localized sample, whereas our sensor measures the integrated column $\mathrm{CO}_{2}$ absorption over a parking lot and woods. Thus depending on the current wind we do not 
expect them to agree completely. However, to first order they should show reasonable correlation if the $\mathrm{CO} 2$ is well mixed.

Figure 9 shows an eight-day correlation of the $\mathrm{CO} 2$ breadboard and the LICOR gas analyzer measure in late Spring of 2007 at the Goddard test range. The agreement, as expected, is not perfect but the two sensors clearly see the same type of variation in the $\mathrm{CO}_{2}$ mixing ratio over an eight-day period. The small gap in the breadboard data was due to a software failure, the sounder ran continuously over this period. The breadboard data are not corrected for temperature.

Error!
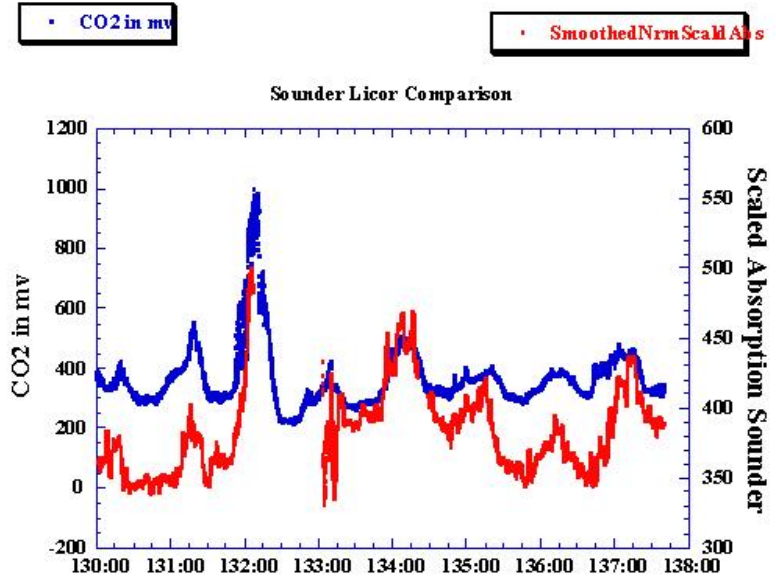

Julian Time (Day:Hour)

Figure 9 is a direct comparison between the lasersounder and a LICOR over eight days of continuous running in late Spring 2007 . The data clearly shows the diurnal cycle. The differences can be attributed to the laser sounder measures a column average while the LICOR is a point measurement.

Figure 10 plots the $\mathrm{CO} 2$ levels at the approach of an early afternoon localised thunderstorm. As the storm approaches the $\mathrm{CO}_{2}$ levels rise, greater absorption, as the air is pushed out in front of the storm. As the storm passes over the measurement path the $\mathrm{CO}_{2}$ levels drop. This was immediately followed by heavy rain which obscured the target and responsible for the increased noise seen in the data. The data is not corrected for temperature or pressure.

We have also integrated a fiber coupled PhotoMultiplier Tube, PMT in to the system. Such detection sensitivity is required due to eye safe intensities consideration which necessitate sensitive detection. A field deployable instrument is currently being commissioned for comparative studies with the $\mathrm{CO} 2$ monitoring facility at the $\mathrm{BAO}$ tower in Erie Colorado. The integration of the PMT is important part of the conversion from a field deployable system to an aircraft instrument.

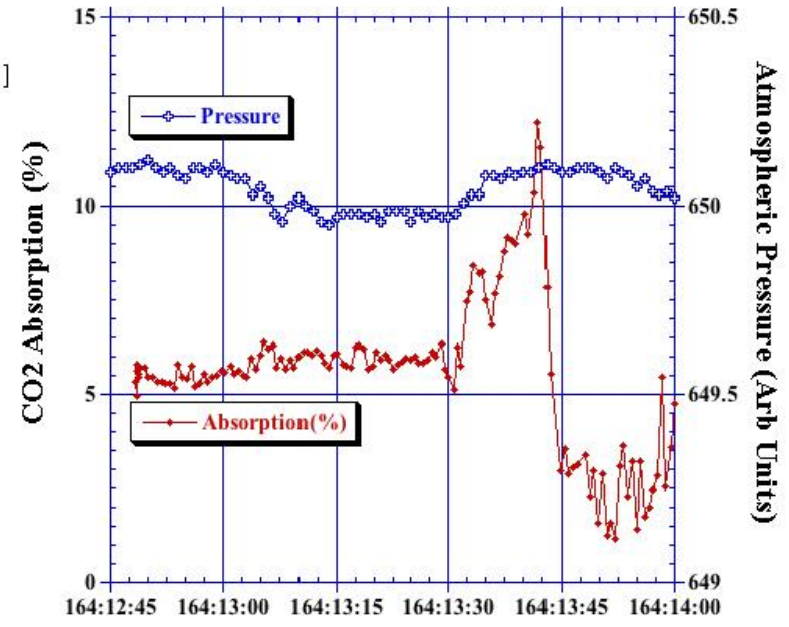

Time (Day, H:M)

Figure 10. Plot of the optical absorption due to $\mathrm{CO} 2$ levels on passage of an early afternoon thunderstorm.

The sounder measured an increase in $\mathrm{CO}_{2}$ as the downdraft from the thunderstorm pushes the air before it into our measurement path. As the storm passes overhead the $\mathrm{CO} 2$ levels drops closely followed by heavy rain ( increased noise). Eventually the rain was so intense as to obscure the target.

Eyan:
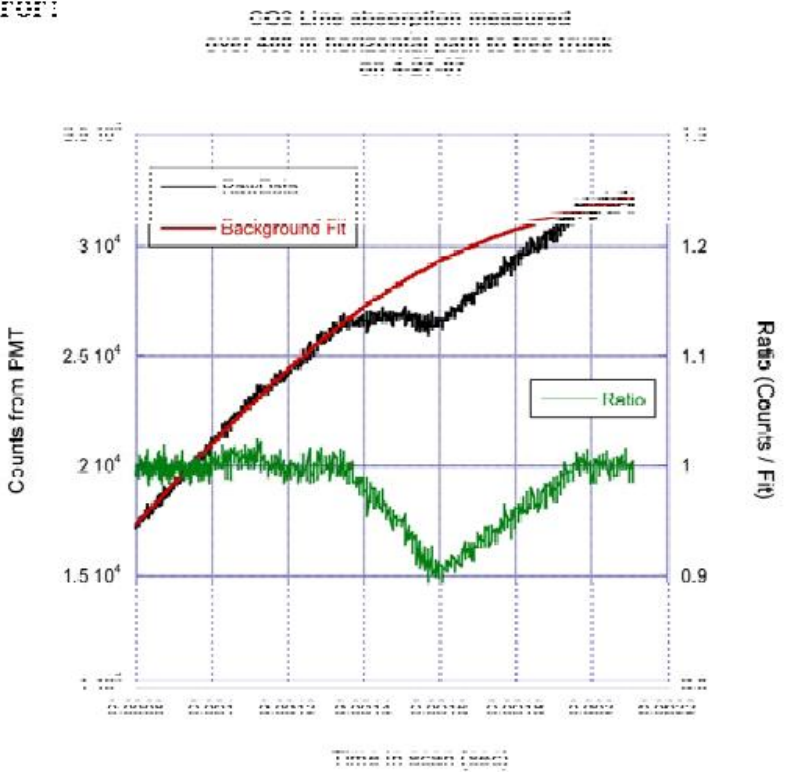

Figure 11. $\mathrm{CO} 2$ absorption at $1572.33 \mathrm{~nm}$ over a $400 \mathrm{~m}$ horizontal path to a diffuse scattering surface (tree trunk), using photon counting PMT. Plotted are the raw counts, background fit and ratio.

Figure 11 shows a typical scan from our initial testing of our fiber coupled PMT. The $\mathrm{CO}_{2}$ absorption is measured with our breadboard system over our open range to an uncooperative target. Data processing for the PMT is nonoptimized but produces a flat response with a clear absorption signature. 


\section{REFERENCES}

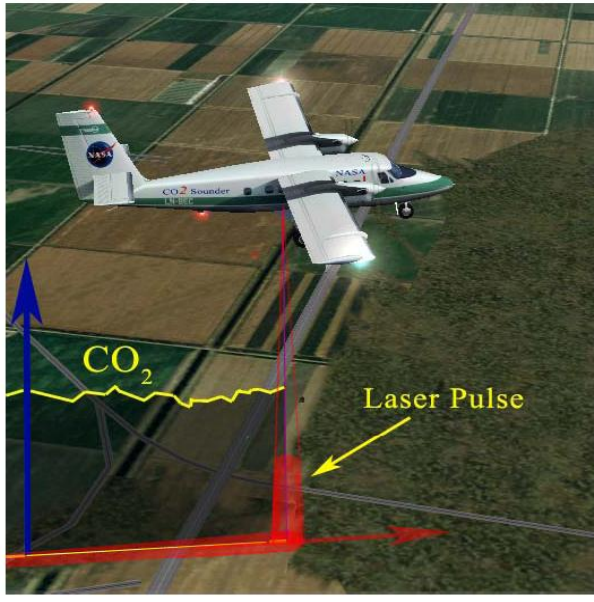

Error!

Figure 12. The next step in development is to deploy an aircraft based $\mathrm{CO}_{2}$ Laser Sounder which measures the column $\mathrm{CO} 2$ from an aircraft to the surface.

\section{SumMary}

Precise satellite observations with adequate temporal and spatial resolution are needed to gain a better understating of the atmospheric $\mathrm{CO}_{2}$ distribution and allow improved modeling of the $\mathrm{CO}_{2}$ cycle. Current estimates indicate that a measurement precision of better than 1 part per million ( 1 ppm) will be needed.

A breadboard $\mathrm{CO}_{2}$ sensor based on erbium fiber amplifiers and diode lasers at $1.57 \mathrm{um}$ has been constructed and tested at NASA GSFC. The breadboard leverages telecommunications component technology and uses solely commercial off-the-shelf components. A combination of hardware and software techniques are applied in order to reduce the effect of various noise sources and other errors drifts (such as etalon fringes) and obtain the required longterm precision. Comparisons with a $\mathrm{CO}_{2}$ measurement standard (LICOR $\mathrm{CO}_{2} / \mathrm{H} 2 \mathrm{O}$ gas analyzer) show very good agreement over an eight-day period. We are currently working on integrating the field deployable instrument with its mobile platform for initial testing at the BAO tower site in Erie, Colorado.

The engineering data from the field tests will help guide repackaging of the laser sounding sensor for airborne experiments, figure 12 , which will demonstrate the needed long term precision and absolute measurement capability from an airborne platform.
[1] D. Crisp, et. al., The Orbiting Carbon Observatory (OCO) mission, Adv. Space Res., 34, (2004), 700-709.

[2] J. Mao and S. R. Kawa, "Sensitivity studies for spacebased measurement of atmospheric total column carbon dioxide using reflected sunlight", Appl. Opt., 43, (2004), 914-92.

[3] P. Werle, R. Miicke, F. Slemr, The Limits of Signal Averaging in Atmospheric Trace-Gas Monitoring by Tunable Diode-Laser Absorption Spectroscopy (TDLAS), Appl. Phys. B 57, 131-139 (1993).

[4] D. T. Cassidy and J. Reid, Atmospheric pressure monitoring of trace gases using tunable diode lasers, App. Phys. B Vol. 29, 279-285 (1982).

[5] J. A. Silver and A. C. Stanton, "Optical interference fringe reduction in laser absorption experiments," Appl. Opt. 27, 1914-1916, 1988.

[6] C. R. Webster, "Brewster-plate spoiler: a novel method for reducing the amplitude of interference fringes that limit tunable-laser absorption sensitivities," J. Opt. Soc. Am. B 2, 1464-1470, 1985.

[7] D.S. Bomse and D.J. Kane, An adaptive singular value decomposition (SVD) algorithm for analysis of wavelength modulation spectra, Appl. Phys. B 85, 461466 (2006).

[8] H. Riris, C. B. Carlisle, R. E. Warren, and D. E. Cooper, Signal-to-noise ratio enhancement in frequencymodulation spectrometers by digital signal processing, Optics Letters, Vol. 19, No. 2, January 15, 1994.

[9] P.W. Werle, P. Mazzinghi a, F. D'Amatoa, M. De Rosa a, K. Maurer, F. Slemr, Signal processing and calibration procedures for in situ diode-laser absorption spectroscopy, Spectrochimica Acta Part A 60 (2004) $1685-1705$.

\section{BIOGRAPHY}

Author biographys unavailable at the time of press. 